\title{
CONVERGENCE OF ISHIKAWA ITERATES OF GENERALIZED NONEXPANSIVE MAPPINGS
}

\author{
M. K. GHOSH \\ Department of Mathematics \\ Narajole Raj College, Midnapore, 721211 \\ West Bengal, INDIA \\ and \\ L. DEBNATH \\ Department of Mathematics \\ University of Central Florida \\ Orlando, Florida, 32816, U.S.A.
}

(Received May 15, 1995 and in revised form September 30, 1996)

\begin{abstract}
This paper is concerned with the convergence of Ishikawa iterates of generalized nonexpansive mappings in both uniformly convex and strictly convex Banach spaces. Several fixed point theorems are discussed.
\end{abstract}

KEY WORDS AND PHRASES. Fixed point theorems, quasi-nonexpansive mappings, uniformly convex Banach spaces, and strictly convex Banach spaces.

1991 AMS SUBJECT CLASSIFICATION CODES. 47H10, 54H25.

\section{INTRODUCTION}

Recently, Hardy and Rogers (1973) discussed the convergence of Ishikawa (1974) iterates of generalized nonexpansive mappings in a metric space These mappings have also been discussed in a Banach space by Goebel et al. (1973), Wong (1976) and Shimi (1978).

The purpose of this paper is to discuss the convergence of the Ishikawa iterates of generalized nonexpansive mappings in a uniformly convex space and in a strictly convex Banach space It is shown that results proved by Ghosh (1990) for nonexpansive mappings are also true for generalized nonexpansive mappings. Furthermore, it is shown that a result of Wong (1976) for the case of Mann iterates (1953) is also true for the case of Ishikawa iterates.

\section{GENERALIZED NONEXPANSIVE MAPPINGS AND ISHIKAWA ITERATIVE PROCESS}

Suppose $B$ is a Banach space and $D$ is a convex subset of $B$. A mapping $T: D \rightarrow D$ is said to be generalized nonexpansive if

$$
\|T x-T y\| \leq a\|x-y\|+b\{\|x-T x\|+\|y-T y\|\}+c\{\|x-T y\|+\|y-T x\|\}
$$

for all $x, y \in D$, where $a, b, c \geq 0$ and

$$
(a+2 b+2 c) \leq 1 .
$$

In this connection we recall a fixed point theorem due to Goebel $e t$ al. (1973), an analogue of which has been established by Browder (1965) and Kirk (1965). 
THEOREM 2.1 (Goebel et al. (1973)) If $D$ is a closed bounded convex subset of a uniformly convex Banach space $B$, and $T: D \rightarrow D$ is a continuous mapping that satisfies (2.1) and (2.2), then $T$ has a fixed point.

REMARK 1. If $b>0$, then the fixed point is unique. For, if possible, suppose $p$ and $q$ are two fixed points of $T$. Then it is easy to derive from (2.1) that

$$
\|p-q\| \leq(a+2 c)\|p-q\|<(a+2 b+2 c)\|p-q\|
$$

which implies that $p=q$.

REMARK 2. A generalized nonexpansive mapping $T$ is also quasi-nonexpansive if it has a fixed point. If $p$ is a fixed point of $T$, then

$$
\|T x-p\| \leq\left(\frac{a+b+c}{1-b-c}\right)\|x-p\| \leq\|x-p\|
$$

because of the fact that $(1-b-c) \geq(a+b+c)$. Thus $T$ is quasi-nonexpansive. This was introduced by Bose and Mukherjee (1981).

REMARK 3. If $D$ is a closed bounded convex subset of a uniformly convex Banach space $B$ and $T: D \rightarrow D$ is a continuous mapping satisfying (2.1) with (2.2), then $T$ is asymptotically regular. The proof of this result may be established exactly in the same way as it has been done by Ghosh (1990).

Suppose $D$ is a convex subset of a Banach space $B$ and $T$ is a self-mapping of $D$. For an $x_{0} \in D$, we define a sequence $\left\{x_{n}\right\}_{n=1}^{\infty}$ such that

$$
x_{n}=T_{\lambda, \mu}^{n} x_{0}, \quad T_{\lambda, \mu}=(1-\lambda) I+\lambda T[(1-\mu) I+\mu T] .
$$

The interative scheme (2.5) was introduced by Ishikawa (1974). Or, in earlier notation,

$$
T_{\lambda, \mu}=(1-\lambda) I+\lambda T T_{\mu},
$$

where $\lambda \in(0,1)$ and $\mu \in[0,1)$, so that $T_{\lambda, \mu}=T_{\lambda}$ when $\mu=0$.

\section{CONVERGENCE OF ISHIKAWA ITERATES}

First, we observe that $F(T)=F\left(T_{\lambda, \mu}\right)$ where $F(T)$ denotes the fixed point set of the generalized nonexpansive mapping $T$. It is obvious that

$$
F(T) \subset F\left(T_{\lambda, \mu}\right) .
$$

If $p \in F\left(T_{\lambda, \mu}\right)$, that is, $T_{\lambda, \mu} p=p$, which implies that $T T_{\mu} p=p$, since $T$ is generalized nonexpansive We then have

$$
\|T p-p\|=\left\|T p-T T_{\mu} p\right\| \leq a\left\|p-T_{\mu} p\right\|+b\left[\|p-T p\|+\left\|p-T_{\mu} p\right\|\right]+c\left\|T p-T_{\mu} p\right\|
$$

The above result gives

$$
(1-b-c)\|T p-p\| \leq(a+b+c)\left\|T_{\mu} p-p\right\|=\mu(a+b+c)\|T p-p\| .
$$

This implies that

$$
\|T p-p\| \leq\left(\frac{\mu(a+b+c)}{1-b-c}\right)\|T p-p\|
$$

whence, we get $T p=p$, since $\left(\frac{\mu(a+b+c)}{1-b-c}\right)<1$. Then

$$
F\left(T_{\lambda, \mu}\right) \subset F(T) \text {. }
$$

Thus (3.1) and (3.3) yield $F(T)=F\left(T_{\lambda, \mu}\right)$.

We now state the following variants of theorems due to Ghosh (1990) and they can be proved by following Ghosh's paper. 
THEOREM 3.1. If $D$ is a bounded closed convex subset of a uniformly convex Banach space $B$ and $T: D \rightarrow D$ be a continuous mapping satisfying (2.1) with (2.2), then $T_{\lambda, \mu}$ is asymptotically regular.

It may be noted that the case, when $\mu=0$ in the above theorem, has been proved by Wong (1976).

THEOREM 3.2. Suppose $D$ is a bounded closed convex subset of a uniformly convex Banach space $B$, and $T: D \rightarrow D$ is a continuous mapping which satisfies (2.1) with (2.2). If $T$ satisfies any one of the following conditions:

(a) ( $\left.I-T T_{\mu}\right)$ maps closed sets in $D$ into closed sets in $B$,

(b) $T T_{\mu}$ is demicompact at $\theta$, then, for an $x_{0} \in D$, the sequence $\left\{x_{n}\right\}$ with $x_{n}=T_{\lambda, \mu}^{n} x_{0}$ converges to a fixed point of $T$ in $D$.

THEOREM 3.3. If $D$ is a bounded closed convex subset of a uniformly convex Banach space $B$ and $T: D \rightarrow D$ be a continuous mapping satisfying (2.1) with (2.2). If there exists a number $k>0$ such that, for each $x \in D$,

$$
\left\|\left(I-T T_{\mu}\right) x\right\| \geq k d(x, F)
$$

then, for an $x_{0} \in D$, the sequence $\left\{x_{n}\right\}$ with $x_{n}=T_{\lambda, \mu}^{n} x_{0}$ converges to a fixed point of $T$ in $D$.

DEFINITION 3.1. A Banach space is said to be strictly convex provided that if

$$
\|x+y\|=\|x\|+\|y\| \quad \text { and } \quad x \neq 0, \quad y \neq 0, \quad \text { then } \quad y=t x, \quad t>0 .
$$

We now prove a result which is close in spirit with the one proved by Wong [4] in connection with the convergence of Mann iterates.

THEOREM 3.4. Suppose $D$ is a compact convex subset of a strictly convex Banach space $B$ and $T: D \rightarrow D$ is a continuous mapping satisfying (2.1) with (2.2). Then, for an $x_{0} \in D$, the sequence $\left\{x_{n}\right\}$ with $x_{n}=T_{\lambda, \mu}^{n} x_{0}$ converges to a fixed point of $T$ in $D$.

PROOF. First, we observe that $F(T)$ is nonempty, because the existence of a fixed point is ensured by the Schauder-Tychonoff theorem. Then, obviously, $F(T)=F\left(T_{\lambda, \mu}\right) \neq \emptyset$. Since $D$ is compact, the sequence $\left\{x_{n}\right\}$ has a subsequence $\left\{x_{n}\right\}$ which converges to a point $x$, say, in $D$. We show that $x$ is a fixed point of $T$. The quasi-nonexpansiveness of $T$ follows from [10]. If $T$ is quasi-nonexpansive, then $T_{\lambda, \mu}$ is also quasi-nonexpansive, as seen from (3.6) below. From the quasi-nonexpansiveness of $T_{\lambda, \mu}$ we observe that the sequence $\left\{\left\|x_{n}-p\right\|\right\}$ is non-increasing, where $p$ is a fixed point of $T$. In fact,

$$
\left\|x_{n+1}-p\right\|=\left\|T_{\lambda, \mu}^{n+1} x_{0}-p\right\| \leq\left\|T_{\lambda, \mu}^{n} x_{0}-p\right\|=\left\|x_{n}-p\right\| .
$$

Now, if $F(T)$ is a singleton, then $p=x$ and the theorem is proved. We now, assume otherwise. Then from the continuity of the norm, $\|\cdot\|$ and $T_{\lambda, \mu}$ we obtain

$$
\begin{aligned}
\|x-p\| & =\lim _{\jmath \rightarrow \infty}\left\|x_{n_{j+1}}-p\right\| \\
& =\lim _{j \rightarrow \infty}\left\|T_{\lambda, \mu}^{n_{\jmath+1}} x_{0}-p\right\| \leq \lim _{\jmath \rightarrow \infty}\left\|T_{\lambda, \mu}^{1+n,} x_{0}-p\right\| \\
& =\lim _{\jmath \rightarrow \infty}\left\|T_{\lambda, \mu} x_{n}-p\right\|=\left\|T_{\lambda, \mu} x-p\right\| .
\end{aligned}
$$

Therefore, because of the quasi-nonexpansiveness of $T_{\lambda, \mu}$ and (3 6), we have

$$
\left\|T_{\lambda, \mu} x-p\right\|=\|x-p\| \text {. }
$$


Indeed,

$$
\begin{aligned}
\left\|T_{\lambda, \mu} x-p\right\| & =\left\|(1-\lambda) x+\lambda T T_{\mu} x-p\right\| \\
& =\left\|(1-\lambda)(x-p)+\lambda\left(T T_{\mu} x-p\right)\right\| \\
& \leq(1-\lambda)\|x-p\|+\lambda\left\|T T_{\mu} x-p\right\| \\
& \leq(1-\lambda)\|x-p\|+\lambda\left\|T_{\mu} x-p\right\| \\
& =(1-\lambda)\|x-p\|+\lambda\|(1-p) x+\mu T x-p\| \\
& =(1-\lambda)\|x-p\|+\lambda\|(1-\mu)(x-p)+\mu(T x-p)\| \\
& \leq(1-\lambda)\|x-p\|+\lambda\|x-p\| \\
& =\|x-p\| .
\end{aligned}
$$

If we take into account (3.7) and (3.8), it follows that inequalities involved in (3.8) reduce to equalities. Thus we have the following results:

$$
\begin{gathered}
\left\|(1-\lambda)(x-p)+\lambda\left(T T_{\mu} x-p\right)\right\|=(1-\lambda)\|x-p\|+\lambda\left\|T T_{\mu} x-p\right\| \\
\left\|T T_{\mu} x-p\right\|=\|x-p\| .
\end{gathered}
$$

Since the space $B$ is strictly convex, then for some $t>0$, we have

$$
T T_{\mu} x-p=t(x-p) .
$$

From (3.10) we observe that $t=1$, which implies that $T T_{\mu} x=x$. Thus $x$ is a fixed point of $T_{\lambda, \mu}$ and hence of $T$. But we have already observed that $\left\{\left\|x_{n}-x\right\|\right\}$ is nonincreasing and hence $\left\{x_{n}\right\}$ converges to $x$. This completes the proof.

REMARK 4. The present analysis can be extended to a more general mapping $T$ which satisfies

$$
\|T x-T y\| \leq \max \left\{\|x-y\|, \frac{1}{2}[\|x-T x\|+\|y-T y\|], \frac{1}{2}[\|x-T y\|+\|y-T x\|]\right\},
$$

for all $x, y \in D$. This mapping includes nonexpansive and generalized nonexpansive mappings (see Rhoades (1977)). It is easy to verify that $T$ is quasi-nonexpansive.

ACKNOWLEDGEMENT. The first author is indebted to Prof. M. Maiti for his suggestion in the preparation of this paper.

\section{REFERENCES}

BOSE, R. K. and MUKHERJEE, R. N., Approximating fixed points of some mappings, Proc. Amer. Math. Soc. 82 (1981), 603-606.

BROWDER, F. E., Nonexpansive nonlinear operations in Banach space, Proc. Nat. Acad. Sci. U.S.A. 54 (1965), 1041-1044.

GHOSH, M. K., On the convergence of Ishikawa iterates to fixed points, Ph.D. thesis, Jadavpur University, India, 1990

GOEBEL, K., KIRK, W. A. and SHIMI, T. N., A fixed point theorem in uniformly convex Banach spaces, Bull. U.M.I. 7 (1973), 67-75.

HARDY, G. F. and ROGERS, T. D., A generalization of a fixed point theorem of Reich, Cand. Math. Bull. 16 (1973), 201-206.

ISHIKAWA, S., Fixed points by a new iteration method, Proc. Amer. Math. Soc. 44 (1974), 147-150

KIRK, W A., A fixed point theorem for mappings which do not decrease distances, Amer. Math. Monthly 72 (1965), 1004-1006.

MANN, W. R., Mean value methods in iteration, Proc. Amer. Math. Soc. 4 (1953), 506-510

RHOADES, B. E., A comparison of various definitions of contractive mappings, Trans. Amer. Soc. 226 (1977), 257-290.

SHIMI, T N., Approximation of fixed points of certain nonlinear mappings, J. Math. Anal. and Appl. 65 (1978), 565-571

WONG, C. S., Approximation of fixed points of generalized nonexpansive mappings, Proc. Amer. Math. Soc. 54 (1976), 93-97. 


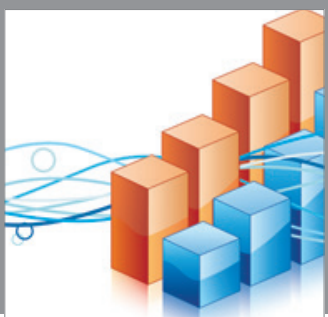

Advances in

Operations Research

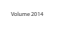

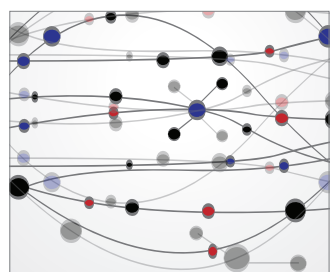

\section{The Scientific} World Journal
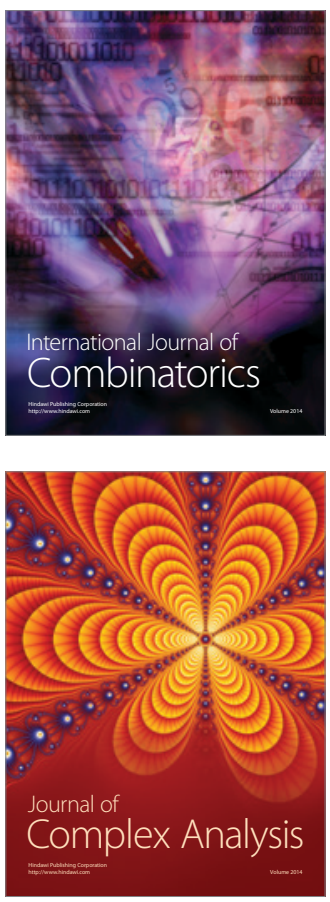

International Journal of

Mathematics and

Mathematical

Sciences
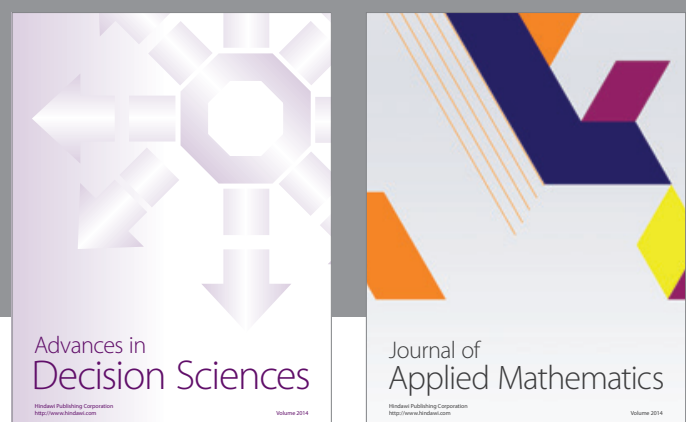

Journal of

Applied Mathematics
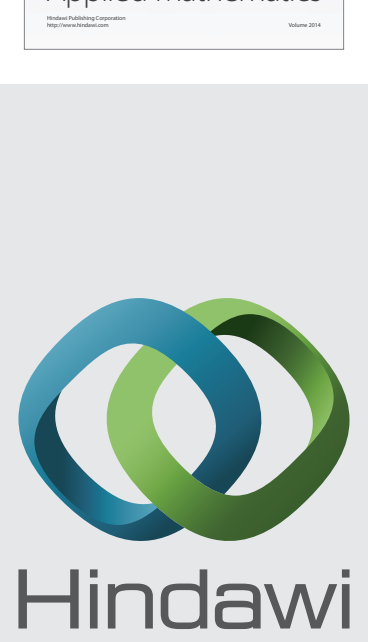

Submit your manuscripts at http://www.hindawi.com
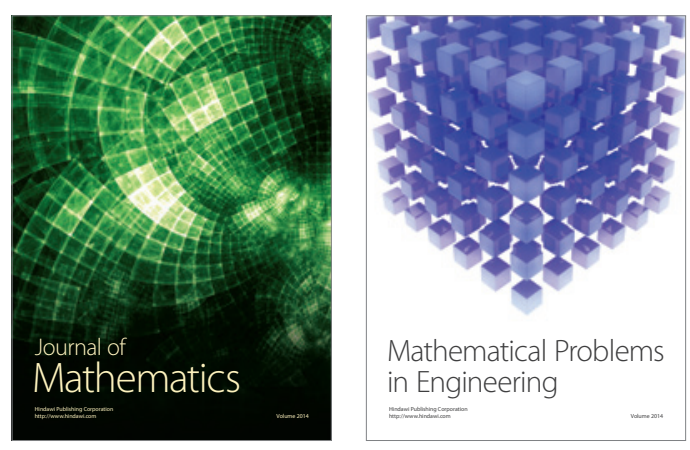

Mathematical Problems in Engineering
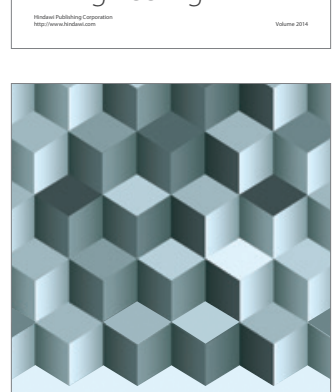

Journal of

Function Spaces
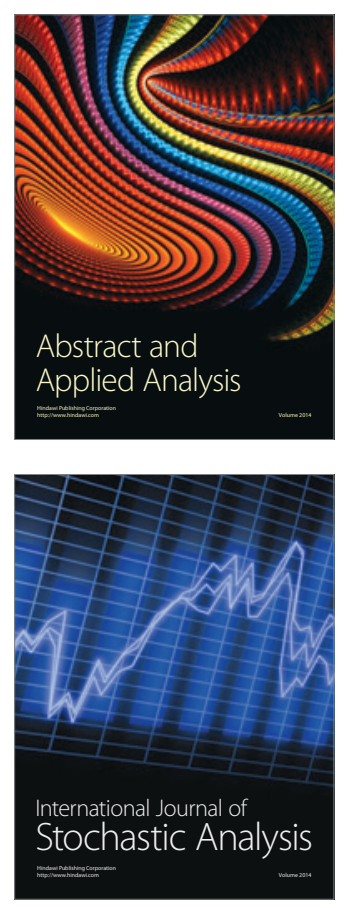

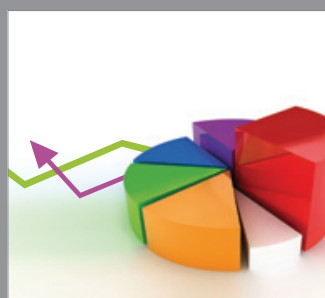

ournal of

Probability and Statistics

Promensencen
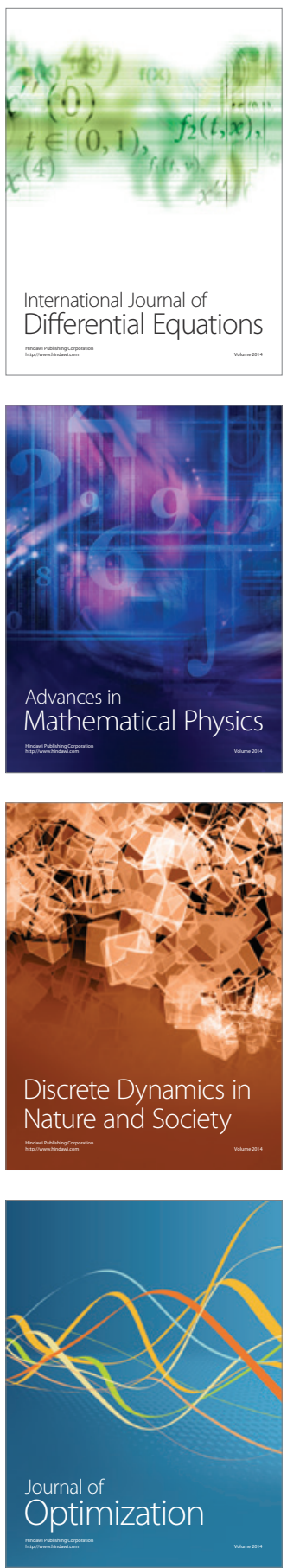